ANL-HEP-PR-03-029

EFI-03-18

FERMILAB-PUB-03/066-T

\title{
Precision Electroweak Data and Unification of Couplings in Warped Extra Dimensions
}

\author{
Marcela Carena ${ }^{a}$, Antonio Delgado $^{b}$, Eduardo Pontón $^{a}$, \\ Tim M.P. Tait ${ }^{a}$, and C.E.M. Wagner ${ }^{c, d}$ \\ ${ }^{a}$ Fermi National Accelerator Laboratory, P.O. Box 500, Batavia, IL 60510, USA \\ ${ }^{b}$ Department of Physics and Astronomy, Johns Hopkins University, \\ 3400 North Charles St., Baltimore, MD 21218, USA \\ ${ }^{c}$ HEP Division, Argonne National Laboratory, 9700 Cass Ave., Argonne, IL 60439, USA \\ ${ }^{d}$ Enrico Fermi Institute, Univ. of Chicago, 5640 Ellis Ave., Chicago, IL 60637, USA
}

November 7, 2018

\begin{abstract}
Warped extra dimensions allow a novel way of solving the hierarchy problem, with all fundamental mass parameters of the theory naturally of the order of the Planck scale. The observable value of the Higgs vacuum expectation value is red-shifted, due to the localization of the Higgs field in the extra dimension. It has been recently observed that, when the gauge fields propagate in the bulk, unification of the gauge couplings may be achieved. Moreover, the propagation of fermions in the bulk allows for a simple solution to potentially dangerous proton decay problems. However, bulk gauge fields and fermions pose a phenomenological challenge, since they tend to induce large corrections to the precision electroweak observables. In this article, we study in detail the effect of gauge and fermion fields propagating in the bulk in the presence of gauge brane kinetic terms compatible with gauge coupling unification, and we present ways of obtaining a consistent description of experimental data, while allowing values of the first Kaluza Klein mode masses of the order of a few $\mathrm{TeV}$.
\end{abstract}




\section{Introduction}

Large extra dimensions have been introduced as a way of solving the hierarchy problem, related to the hierarchy between the Planck and the weak scales. In flat extra dimensions, this can be achieved by assuming that the Standard Model (SM) fields are localized on a four dimensional brane, while gravity propagates in the extra dimensional bulk [1]. Under such conditions, the fundamental Planck scale may be of order of the weak scale. The weakness of the gravitational interactions is explained by the large volume suppression of the zero mode graviton interactions with the localized SM fields. For this mechanism to work, the size of the extra dimensions must be several orders of magnitude larger than the fundamental scale of the model.

An interesting alternative is achieved in the case of warped extra dimensions proposed by Randall and Sundrum (RS) [2]. In this case, one can obtain a solution to the hierarchy problem by assuming that the gravity field propagates in the bulk of a slice of 5-dimensional anti de Sitter space $\left(\mathrm{AdS}_{5}\right)$ bounded by 4-dimensional "branes", while the Higgs field is localized on the brane where the warp factor is small (the $\mathrm{TeV}$ or IR brane). In this case, all mass parameters, the fundamental Planck scale, the curvature and the size of the extra dimensions, as well as the Higgs vacuum expectation value (VEV), are of the same order. Due to the nontrivial warp factor, however, the observable Higgs VEV is red-shifted to values much smaller than the fundamental Planck scale.

In the minimal model of warped extra dimensions only gravity propagates in the bulk, while the SM gauge and fermion fields are localized on the same brane as the Higgs field. The solution to the hierarchy problem, however, demands only the localization of the Higgs field, and there is strong motivation to consider the propagation of the gauge fields in the bulk of the extra dimension. In particular, at scales below the AdS curvature $k$, the gauge couplings evolve logarithmically [3, 4, and unification of couplings in these scenarios may be naturally achieved whenever the gauge fields propagate in the bulk [5] (for a related discussion in a supersymmetric context, see [6]). Bulk gauge fields are, however, phenomenologically challenging since they tend to induce large corrections to the precision electroweak observables. These corrections are induced by a combination of two effects: for one, the presence of a localized Higgs VEV induces a repulsion of the zero mode of the gauge fields from the brane location, implying a modification of the weak gauge boson couplings to the brane fields and of the relation between the tree-level weak gauge boson masses and the Higgs VEV. Since these Higgs localization effects are absent for the photon field, and are different for the $W$ and 
the $Z$ gauge bosons, this induces important tree-level corrections to the precision electroweak observables [7, 8. Furthermore, the Kaluza-Klein (KK) modes of the gauge fields couple to the brane fields with a coupling which is $\sqrt{2 k L}$ times larger than the zero mode gauge coupling, where $L$ is the proper size of the extra dimension. The dimensionless factor $k L$ has to be about 30 so that the Higgs VEV is red-shifted from the Planck to the TeV scale. Therefore, the KK modes couple strongly to the brane quark and lepton fields and can induce large effects on the precision electroweak observables.

The above effects imply a lower bound on the mass of the lightest KK mode of the gauge fields of the order of $27 \mathrm{TeV}$ [8]. Such a large bound excludes the possibility of direct detection of these KK modes at the Tevatron and the LHC. It also reintroduces to some extent the hierarchy problem that the RS scenario intended to solve. However, in Refs. [9, 10] it was shown that these bounds may be relaxed by considering the possible effect of brane localized kinetic terms for the gauge fields [1]. Large brane kinetic terms have the effect of enhancing the four dimensional properties of the gauge fields as seen by brane observers. In particular, the presence of gauge kinetic terms on the IR brane, where the Higgs and fermion fields are localized, translates into a reduction of the Higgs localization effects, as well as of the effective coupling of the heavy KK modes with respect to the one of the zero modes. In addition, the KK masses depend strongly on the IR brane kinetic term and can decrease by up to a factor of ten when these terms are large (and positive). As a result, the bound on the mass of the lightest gauge KK mode mass may be relaxed down to a few $\mathrm{TeV}$. Moreover, if the heavy flavor Z-pole observables are excluded from the data, it was found that, for certain values of the weak gauge boson KK masses and of the local gauge kinetic terms, the fit to the precision electroweak observables improves with respect to the one obtained within the Standard Model with no extra dimensions.

In this work we are interested in considering the scenario of warped extra dimensions in the light of grand unification. The above described scenario, with the lightest KK modes in the few $\mathrm{TeV}$ range, requires large brane kinetic terms on the IR brane. However, we will find that it is necessary to assume that the GUT symmetry is broken on the IR brane, which implies that the local kinetic terms need not be unified. Indeed, generically the brane kinetic terms include bare contributions, which encode the physics at or above the cutoff scale, as well as radiative contributions that are calculable within the effective 5-dimensional theory. On the IR brane, the latter effects are small since the local cutoff is not much above the TeV scale and there is no room for a large logarithmic enhancement. Hence, a large IR brane kinetic term 
would have to arise from the unknown UV physics and it becomes necessary to assume that this physics respects the GUT symmetry. The situation is different for the UV brane kinetic terms since there the local cutoff is of the order of the Planck scale and the loop contributions are logarithmically enhanced at low energies. In fact, the large GUT violating logarithms that are found in the calculation of the low-energy gauge couplings [3] can be understood as arising from the RG flow of the UV brane kinetic terms, from the Planck to the TeV scale 44. On the other hand, most models of warped unification seem to require orbifold breaking of the gauge symmetry. Thus, to retain the predictivity of these grand unified scenarios (e.g. for the Weinberg angle), it is necessary to argue that the bare contributions to the brane kinetic terms are small. One such argument is furnished by naive dimensional analysis (NDA) [12], which assumes strong coupling at the cut-off scale and demands brane terms to be small. Nevertheless, it should be kept in mind that the IR kinetic terms could be larger than what naive considerations would suggest, and have important effects for the spectrum of lowest lying KK modes.

A further complication comes from the fact that the RS unified model has TeV mass KK gauge bosons which can mediate proton decay. In fact, since the effective cut-off on the IR brane is $\mathrm{TeV}$, even non-renormalizable operators can lead to unacceptably short proton lifetimes. These problems may be alleviated by assuming that the quarks and leptons come from different multiplets, and their partners are projected out by particular boundary conditions. Clearly, this idea can only work if fermions also propagate in the bulk. In principle, fermions propagating in the bulk tend to improve the two phenomenological challenges associated with bulk gauge fields, since these are mostly related to the localization of the fermion fields in the same brane as the Higgs field. However, it has been suggested that bulk fermions do not lead to a dramatic relaxation of these constraints [13]. Moreover, the bulk fermions induce new contributions to the precision electroweak observables [14], which can only be suppressed if at least some of the third generation fermions are localized close to the infrared brane.

In this article, we shall analyze all these problems in detail and we shall demonstrate that KK mode masses of the order of several TeV can be achieved by assuming either a non-vanishing IR gauge kinetic term for the gauge fields, or a large Higgs mass, together with a particular geography of the fermion fields in the extra dimensional bulk. In section 2, we describe the model and introduce the question of unification. In section 3 we present a formalism to study the experimental implications in a simple and straightforward way. In section 4, we analyze the novel effects to precision electroweak observables due to the Higgs localization effects and 
the contribution of the KK tower of gauge bosons and fermions in the bulk. In section 5, we discuss the fit to the precision electroweak data. We reserve section 6 for our conclusions.

\section{Grand Unification}

In this section we review the important features of grand unification in RS. While we envision the non-supersymmetric model of Ref. [5], our framework is quite general, defined by the low energy theory, and thus we expect our conclusions to hold more generally for the RS unified framework. The background metric is defined by the line element,

$$
d s^{2}=G_{M N} d X^{M} d X^{N}=e^{-2 \sigma} \eta_{\mu \nu} d x^{\mu} d x^{\nu}+d y^{2}
$$

where $X^{M}=\left(x^{\mu}, y\right)$ denote the 5-dimensional coordinates, $\eta_{\mu \nu}=\operatorname{diag}(-1,+1,+1,+1), \sigma(y)=$ $k|y|$ and $0 \leq y \leq L$. The gauge bosons are assumed to propagate in the bulk, while the standard model Higgs is localized on the IR brane at $y=L$. We will also assume that the fermions propagate in the bulk since in this case the question of proton decay can be more naturally addressed, as we discuss below.

An important issue is how the grand unification group is broken to the standard model group. In the higher dimensional context there are various possibilities. These include dynamical symmetry breaking through the Higgs mechanism (as in 4-dimensional models), as well as intrinsically higher dimensional mechanisms where the gauge symmetry is broken by nontrivial boundary conditions [15]. Orbifold breaking seems to be a powerfull ingredient in the building of realistic models. When the symmetry is broken by orbifold boundary conditions, the GUT symmetry is not an exact symmetry of the theory since there are special points in spacetime where the symmetry is reduced to a smaller group. Operators living on the special points are generally not GUT-symmetric, and can spoil the GUT predictions. In particular, it is necessary to make further assumptions about the size of the localized terms that need not respect the GUT symmetry. One way to proceed is based on NDA, which assumes that all couplings get strong at the cut-off scale of the effective 5-dimensional theory. This provides a well defined framework in which the symmetry violating terms are sufficiently suppressed to be able to discuss unification quantitatively.

Let us emphasize that in order to achieve unification in the non-supersymmetric case, relevant threshold corrections at the grand unification scale must be present. In warped extra dimensions, these corrections arise naturally if the gauge symmetry is broken by bulk Higgs 
VEV's [5]. As we will discuss below, for light KK boson masses to be compatible with present phenomenological constraints, the GUT symmetry also needs to be broken by orbifold boundary conditions on the IR brane. Thus, in this work, we shall assume that the GUT symmetry is broken by a bulk Higgs VEV, as well as by orbifold boundary conditions on the IR brane.

The dependence of the 3-2-1 gauge couplings measured by low energy observers (at energy scales $q \ll k e^{-k L}$ ) on the GUT scale in this type of compactifications has been studied in previous articles [3, 4], and it was found that, contrary to what happens in flat extra dimensions, the differences between low-energy couplings depend logarithmically on the fundamental scales of the theory and are given by:

$$
\frac{1}{g_{i}^{2}(q)}-\frac{1}{g_{j}^{2}(q)}=\left(\frac{1}{g_{U V}^{i^{2}}}-\frac{1}{g_{U V}^{j^{2}}}\right)+\left(\frac{1}{g_{I R}^{i^{2}}}-\frac{1}{g_{I R}^{j^{2}}}\right)+\frac{\left(b_{i}-b_{j}\right)}{8 \pi^{2}} \log \frac{k}{q}+\left(\Delta_{i}-\Delta_{j}\right) k L+\mathcal{O}(1) .
$$

Here the first two terms can be thought of as the "bare" values of the localized couplings, while the log term arises from loop effects and is identical to the contribution that is found in purely 4-dimensional theories. The bulk couplings get renormalized in a universal manner and, apart from threshold corrections, cancel in the differences. The $\Delta_{i}$ terms correspond to possible threshold corrections arising from the GUT breaking VEV's of bulk Higgs fields, and have the important property of being enhanced by $k L[5]$. Finally, the $\mathcal{O}(1)$ represents further subdominant contributions. The leading, GUT violating, logarithmic effects in Eq. (2) can be understood as arising from the RG evolution of the UV brane kinetic terms. It was shown in Ref. [4] that, for $k \gg q \gg k e^{-k L}$,

$$
\frac{1}{g_{U V}^{i^{2}}(q)}=\frac{1}{g_{U V}^{i^{2}}}+\frac{b_{i}}{8 \pi^{2}} \log \frac{k}{q}+\text { GUT symmetric }
$$

which exhibits precisely the large logs found in the low-energy calculation. The precise $\beta$ function coefficients depend on which fields propagate in the bulk and most importantly on whether those fields have a significant overlap with the UV brane. In fact, in the RS scenario the heavy KK modes of all fields are localized towards the IR brane and therefore each KK mode gives only a tiny contribution to the low-energy gauge coupling. Although the sum of all of them can give a contribution as large as the log in Eq. (3), when including the full GUT field content the heavy KK modes give a universal contribution that cancels in the differential "running". Thus, the difference in the 3-2-1 low-energy gauge couplings is effectively determined by the zero modes alone. This is one way to understand the effective four dimensional behavior.

The gauge zero modes always contribute with full strength to $b_{i}$ since their wavefunctions are flat. For the fermions, however, it is possible to add a bulk mass term, $M$, whose effect is to 
control the localization of the zero mode. When $M>k / 2$, the zero mode is localized towards the UV brane and fully contributes to the gauge coupling running. However, when $M<k / 2$ the zero mode is exponentially suppressed at the UV brane and effectively decouples from the running above the $\mathrm{TeV}$ scale. There is also an intermediate region in which the fermions contribute only partially to the renormalization of the gauge coupling. Then, splitting the gauge and fermion contributions, the $\beta$-function coefficients are given by $b_{1}=n_{f, 1}, b_{2}=-22 / 3+n_{f, 2}$ and $b_{3}=-11+n_{f, 3}$, where the $n_{f, i}$ terms are associated with the fermions. Note that if the fermions come in complete multiplets, then they contribute universally: $n_{f, 1}=n_{f, 2}=n_{f, 3}$. However, we have seen that considerations related to proton decay forces us to split them into different multiplets, which can then have different bulk masses. In practice, we will assume universal fermion bulk masses and, as a result, the fermions do not have an impact on whether the gauge couplings unify or not.

It is important to emphasize that within the framework of grand unification in warped extra dimensions, there is always a tower of $X Y$ gauge bosons with masses of order $k e^{-k L} \sim$ $\mathrm{TeV}$, where in general $X Y$ denote the non-standard gauge bosons arising as a remnant of the broken grand unified symmetry. In fact, this is an essential feature which allows one to express the differences in the zero mode couplings, Eq. (2), in terms of large logs of only the four dimensional beta functions. The key point is that the higher KK modes always come in complete GUT multiplets (the heavy KK modes of the broken and unbroken gauge bosons are approximately degenerate in mass) and do not influence the coupling differences in a large way [3. However, this remarkable feature, a clear distinction of the RS-style grand unification from the more conventional 4-dimensional unification scenario, is also a potential problem phenomenologically. If the quarks and leptons are unified in GUT multiplets, these gauge bosons will induce proton decay at an unacceptable rate. One way of avoiding this problem is to invoke boundary conditions that break the GUT symmetry, which implies that some of the components within a given multiplet will get TeV masses. To accommodate the SM spectrum it is then necessary to double the number of bulk matter multiplets, with the result that quarks and leptons reside in different multiplets. For example, for an $S U(5)$ symmetry the zero mode $d_{R}$ and lepton doublet of a given family arise from different $\overline{5}$ 's. Thus, these models do not have a true unification of SM quarks and leptons. On the other hand the dangerous couplings of broken gauge bosons to pairs of zero mode fermions are absent and cannot mediate proton decay. One still has to worry about higher dimension operators. For example, if baryon violating operators are present on the IR brane, they will only be suppressed by the TeV scale. 
Thus, it is necessary to forbid such operators up to a very high order. One possibility is to impose some sort of gauged baryon symmetry, which is broken only on the UV brane [5]. All the generated baryon violating operators will then be suppressed by the Planck scale.

For the analysis of the electroweak constraints, we will concentrate on an effective theory valid just above the $\mathrm{TeV}$ scale, and use the previous considerations to motivate certain relations among the effective parameters. We can then match to an effective 4-dimensional theory and use the well measured low-energy data to fix these parameters.

\section{The 4-dimensional Effective Theory}

We are interested in determining the bounds on the effective compactification scale, $k e^{-k L}$, in the class of models described in the previous section. These arise both from deviations from the standard model in the zero-mode sector of the theory, as well as from the effects mediated by the Kaluza-Klein towers of the various bulk fields. The former are associated with deformations of the zero-mode wavefunctions due to the electroweak breaking Higgs VEV, which is localized on the IR brane. The latter arise from exchange of KK mode gauge bosons or fermions between zero mode fields. The gauge KK towers induce four-fermion operators that can be important in some regions of parameter space. Since these are tree-level effects, they can potentially impose the strongest constraints on the model. In addition, due to the large top Yukawa coupling, the top KK tower can induce important contributions to the $T$ parameter, even though these arise at loop level. Similarly dangerous contributions can arise from the KK modes of the broken gauge bosons. The simplest way to analyze these effects is to first obtain the effective 4-dimensional theory. In this section, we derive the general form of the low-energy theory and determine a region in parameter space where the top loop effects can be neglected.

In order to study the low-energy constraints, as well as the properties of the lowest lying KK modes, we can consider an effective 5-dimensional theory with renormalized parameters at the matching scale $\sim k e^{-k L}$. The effective action describing the standard model sector (i.e. the standard model fields together with their corresponding KK towers) is then given by

$$
\begin{aligned}
S=\int_{0}^{L} d^{4} x d y \sqrt{-G}\{ & -\frac{1}{2 g_{5}^{2}} W_{M N}^{+} W_{-}^{M N}-\frac{1}{4 g_{5}^{2}} W_{M N}^{3} W_{3}^{M N} \\
& \left.-\frac{1}{4 g_{5}^{\prime 2}} B_{M N} B^{M N}+\mathcal{L}_{\Psi}-2 \delta(y) \mathcal{L}_{U V}-2 \delta(y-L) \mathcal{L}_{I R}\right\}
\end{aligned}
$$


with

$$
\begin{aligned}
\mathcal{L}_{U V}= & \frac{1}{2 g_{U V}^{2}} W_{\mu \nu}^{+} W_{-}^{\mu \nu}+\frac{1}{4 g_{U V}^{2}} W_{\mu \nu}^{3} W_{3}^{\mu \nu}+\frac{1}{4 g_{U V}^{\prime 2}} B_{\mu \nu} B^{\mu \nu} \\
\mathcal{L}_{I R}= & \frac{1}{2 g_{I R}^{2}} W_{\mu \nu}^{+} W_{-}^{\mu \nu}+\frac{1}{4 g_{I R}^{2}} W_{\mu \nu}^{3} W_{3}^{\mu \nu}+\frac{1}{4 g_{I R}^{\prime 2}} B_{\mu \nu} B^{\mu \nu} \\
& +v^{2} W_{\mu}^{+} W_{-}^{\mu}+\frac{1}{2} v^{2}\left(W_{\mu}^{3}-B_{\mu}\right)^{2}+\mathcal{L}(h, \Psi)
\end{aligned}
$$

where $W^{ \pm}, W^{3}$ are the $S U(2)$ gauge bosons and $B$ corresponds to the $U(1)_{Y}$ vector, with SM hypercharge normalization. According to the picture discussed in the previous section, and assuming for example a $S U(5)$ grand unified group, the parameters in the action Eq. (4) obey the GUT relations $g_{5}^{\prime} \approx \sqrt{\frac{3}{5}} g_{5}$ and $g_{I R}^{\prime} \approx \sqrt{\frac{3}{5}} g_{I R}$, while $g_{U V}^{\prime}$ and $g_{U V}$ differ by the additional logarithmic terms in Eq. (2). For simplicity, we neglect the threshold corrections $\Delta_{i}$ in Eq. (2), which would contribute to the bulk gauge couplings. In this way, we can capture the leading loop effects arising from bulk fields and may proceed with a tree-level analysis.

The SM Higgs is located on the IR brane, as required for the RS solution to the hierarchy problem. It has a potential which generates a $\operatorname{VEV}\left\langle H^{0}\right\rangle \equiv \sqrt{2} v$ of order $k$, but due to the background warping, the observable VEV appears red-shifted to $\tilde{v}=v e^{-k L} \approx 123 \mathrm{GeV}$. This induces electroweak symmetry breaking, with localized mass terms generated for the electroweak gauge bosons. $\mathcal{L}(h, \Psi)$ contains the Higgs kinetic and potential terms, as well as the Yukawa couplings

$$
\lambda_{u} \tilde{H} \bar{\Psi}_{Q} \Psi_{t_{R}}+\lambda_{d} H \bar{\Psi}_{Q} \Psi_{b_{R}}+\lambda_{e} H \bar{\Psi}_{L} \Psi_{e_{R}}
$$

where $Q=\left(t_{L}, b_{L}\right)$ and $L=\left(\nu_{L}, e_{L}\right)$ denote the $S U(2)$ quark and lepton doublets and $t_{R}, b_{R}$ and $e_{R}$ denote the quark and lepton $S U(2)$ singlets. For simplicity, we have omitted the generation indices. Note that all of these are 5-dimensional fermions that, after a KK decomposition, include both left- and right-handed towers. The $L$ and $R$ subscripts simply indicate which one of these towers contains a zero mode after the orbifold projection. Also, unlike the 4-dimensional SM, the Yukawa coupling matrices $\lambda_{u}, \lambda_{d}$ and $\lambda_{e}$ have mass dimension -1 .

We have not explicitly written the gluon terms, as they are irrelevant for the electroweak constraints. We will discuss the bulk lagrangian for the fermions, $\mathcal{L}_{\Psi}$, which contains the kinetic as well as possible bulk mass terms, below. 


\subsection{Integrating out the Kaluza-Klein tower in the Gauge Sector}

We begin by integrating out the massive Kaluza-Klein modes of the electroweak gauge sector of the theory. It will be sufficient to do so at tree-level. In the presence of the gauge kinetic terms required by unification in the Randall-Sundrum scenario, as well as the localized Higgs VEV, $v$, it is difficult to diagonalize the quadratic part of the gauge action exactly and, as a result, the identification of the massive eigenstates that need to be integrated out is somewhat tricky. However, the electroweak precision measurements require that $v$ be much smaller than the curvature $k$, and it becomes possible to treat the electroweak breaking effects perturbatively. Thus, we can make a KK decomposition assuming that $v=0$, integrate out these "unperturbed" massive modes, and include the effects of $v$ in the low-energy effective theory as a perturbation.

It will be enough to consider the relevant part of the 5-dimensional action, Eq. (4), that describes the $S U(2) \times U(1)$ neutral gauge bosons. It can be written as follows:

$$
S_{N}=\int_{0}^{L} d^{4} x d y\left\{\frac{1}{2} W_{\mu}^{3} \mathcal{O}^{\mu \nu} W_{\nu}^{3}+\frac{1}{2} B_{\mu} \mathcal{O}^{\prime \mu \nu} B_{\nu}+\mathcal{L}_{\psi}-2 \delta(y-L) \frac{1}{2} \tilde{v}^{2}\left(W_{\mu}^{3}-B_{\mu}\right)^{2}\right\},
$$

where $\tilde{v}=v e^{-k L}$,

$$
\mathcal{O}^{\mu \nu} \equiv \frac{1}{g_{5}^{2}}\left[P^{\mu \nu}+\eta^{\mu \nu} \partial_{y}\left(e^{-2 \sigma} \partial_{y}\right)+2 \delta(y) r_{U V} P^{\mu \nu}+2 \delta(y-L) r_{I R} P^{\mu \nu}\right],
$$

and $\mathcal{O}^{\prime \mu \nu}$ is obtained from Eq. (92) by the replacements $g_{5} \rightarrow g_{5}^{\prime}, r_{U V} \rightarrow r_{U V}^{\prime}$ and $r_{I R} \rightarrow r_{I R}^{\prime}$. Here we introduced the quantities $r_{i} \equiv g_{5}^{2} / g_{i}^{2}$ and $r_{i}^{\prime} \equiv g_{5}^{\prime 2} / g_{i}^{\prime 2}$, which have dimensions of length. As explained in the previous section, the assumption of unification implies that (assuming SU(5), for example) $g_{5}^{\prime} \approx \sqrt{\frac{3}{5}} g_{5}$ and $g_{I R}^{\prime} \approx \sqrt{\frac{3}{5}} g_{I R}$, so that $r_{I R}^{\prime} \approx r_{I R}$. The UV brane parameters, $r_{U V}$ and $r_{U V}^{\prime}$ account for the difference in the low-energy couplings of $S U(2)$ and $U(1)$. In Eq. (9) we also defined the transverse operator $P^{\mu \nu} \equiv \eta^{\mu \nu} \partial^{2}-\partial^{\mu} \partial^{\nu}$. For the analysis of the low-energy constraints, it is clearly important to keep track of the fermion-gauge boson interactions,

$$
\mathcal{L}_{\psi} \supset-e^{-3 \sigma} \bar{\Psi} \Gamma^{\mu}\left(T^{3} W_{\mu}^{3}+Y B_{\mu}\right) \Psi
$$

where $\Psi$ denotes a bulk standard model fermion, $T^{3}$ is the third weak iso-spin generator and $Y$ the hypercharge.

We are interested in the effective theory valid for momenta $p \ll k e^{-k L}$, obtained by integrating out the massive KK modes at tree-level. As mentioned above, we can treat the electroweak symmetry breaking VEV as a perturbation. We could then express the action Eq. (8) in terms of the "unperturbed" KK wavefunctions, integrate out the heavy KK states and sum up their 
effects on the gauge zero modes (which will get a mass of order $g^{2} \tilde{v}^{2}$ after including $v$ perturbatively). It is however simpler to treat the heavy KK modes as a single entity by working in the 5-dimensional picture and integrating out the higher dimensional gauge field with the zero-mode subtracted. More precisely, we write the 5-dimensional gauge fields as

$$
\begin{aligned}
W_{\mu}^{3}(x, y) & =g W_{\mu}^{(0)}(x)+\tilde{W}_{\mu}^{3}(x, y) \\
B_{\mu}(x, y) & =g^{\prime} B_{\mu}^{(0)}(x)+\tilde{B}_{\mu}(x, y)
\end{aligned}
$$

and require the orthogonality conditions

$$
\begin{gathered}
\int_{0}^{L} d y W_{\mu}^{(0)} \tilde{W}_{\nu}^{3}\left[1+2 \delta(y) r_{U V}+2 \delta(y-L) r_{I R}\right]=0 \\
\int_{0}^{L} d y B_{\mu}^{(0)} \tilde{B}_{\nu}\left[1+2 \delta(y) r_{U V}^{\prime}+2 \delta(y-L) r_{I R}\right]=0,
\end{gathered}
$$

which ensure that $\tilde{W}_{\mu}$ and $\tilde{B}_{\mu}$ contain exactly the part of the gauge fields with a nonzero "momentum" in the extra dimension (corresponding to the heavy modes in the KK picture). In order to obtain the correct normalization of the zero-modes $W_{\mu}^{(0)}$ and $B_{\mu}^{(0)}$, we factored out explicitly the zero-th order gauge couplings defined by

$$
g^{2}=\frac{g_{5}^{2}}{L+r_{U V}+r_{I R}}, \quad g^{\prime 2}=\frac{g_{5}^{\prime 2}}{L+r_{U V}^{\prime}+r_{I R}} .
$$

It is very convenient to consider the propagator associated with $\tilde{W}_{\mu}, \tilde{B}_{\mu}$, which is given by

$$
\tilde{G}_{\mu \nu}=G_{\mu \nu}-G_{\mu \nu}^{(0)}
$$

where $G_{\mu \nu}$ is the full (unperturbed) gauge propagator and $G_{\mu \nu}^{(0)}$ the propagator for the zeromode. Both quantities on the right-hand-side of Eq. (14) are easily calculated. For example, for $W_{\mu}^{3}$ (in $W_{5}^{3}=0$ gauge), the full propagator in mixed position and momentum space has the form

$$
G_{\mu \nu}^{3}\left(p ; y, y^{\prime}\right)=\frac{P_{\mu \nu}}{p^{2}} G_{p}^{3}\left(y, y^{\prime}\right)-\frac{p_{\mu} p_{\nu}}{p^{2}} G_{0}^{3}\left(y, y^{\prime}\right)
$$

Here $p^{2} \equiv-\eta_{\mu \nu} p^{\mu} p^{\nu}$ is timelike in the physical region and

$$
G_{p}^{3}\left(y, y^{\prime}\right)=-\frac{\pi g_{5}^{2} e^{k\left(y+y^{\prime}\right)}}{k(A D-B C)}\left[A J_{1}\left(\frac{p}{k} e^{k y<}\right)-B Y_{1}\left(\frac{p}{k} e^{k y<}\right)\right]\left[C J_{1}\left(\frac{p}{k} e^{k y>}\right)-D Y_{1}\left(\frac{p}{k} e^{k y>}\right)\right],
$$


where $J_{\alpha}, Y_{\alpha}$ are Bessel functions of order $\alpha, y_{<(>)}$are the smallest (largest) of $y, y^{\prime}$ and

$$
\begin{aligned}
A & =Y_{0}\left(\frac{p}{k}\right)+p r_{U V} Y_{1}\left(\frac{p}{k}\right) \\
B & =J_{0}\left(\frac{p}{k}\right)+p r_{U V} J_{1}\left(\frac{p}{k}\right) \\
C & =Y_{0}\left(\frac{p}{k} e^{k L}\right)-p e^{k L} r_{I R} Y_{1}\left(\frac{p}{k} e^{k L}\right) \\
D & =J_{0}\left(\frac{p}{k} e^{k L}\right)-p e^{k L} r_{I R} J_{1}\left(\frac{p}{k} e^{k L}\right) .
\end{aligned}
$$

The zero-mode propagator, on the other hand is simply given by

$$
G_{\mu \nu}^{(0)}=\frac{g^{2}}{p^{2}} \eta_{\mu \nu} .
$$

Replacing Eq. (11) in the action Eq. (8), we can derive the equations of motion for $\tilde{W}_{\mu}^{3}$ :

$$
\mathcal{O}^{\mu \nu} \tilde{W}_{\nu}^{3}=e^{-3 \sigma} \bar{\Psi} \Gamma^{\mu} T^{3} \Psi+2 \delta(y-L) \tilde{v}^{2}\left[\left(g W_{(0)}^{\mu}-g^{\prime} B_{(0)}^{\mu}\right)+\tilde{W}_{3}^{\mu}-\tilde{B}^{\mu}\right] .
$$

The classical solution to this equation can be written with the help of the "KK propagator", Eq. (14), as

$$
\begin{aligned}
\tilde{W}_{\mu}^{3}(X) & =\int d X^{\prime} \tilde{G}_{\mu \nu}^{3}\left(X, X^{\prime}\right)\left\{e^{-3 \sigma} \bar{\Psi} \Gamma^{\nu} T^{3} \Psi+2 \delta(y-L) \tilde{v}^{2}\left[\left(g W_{(0)}^{\nu}-g^{\prime} B_{(0)}^{\nu}\right)+\tilde{W}_{3}^{\nu}-\tilde{B}^{\nu}\right]\right\}\left(X^{\prime}\right) \\
& \approx \int d X^{\prime} \tilde{G}_{\mu \nu}^{3}\left(X, X^{\prime}\right)\left\{e^{-3 \sigma} \bar{\Psi} \Gamma^{\nu} T^{3} \Psi+2 \delta(y-L) \tilde{v}^{2}\left[g W_{(0)}^{\nu}-g^{\prime} B_{(0)}^{\nu}\right]\right\}\left(X^{\prime}\right),
\end{aligned}
$$

where $X=\left(x^{\mu}, y\right)$, and in the second line we kept terms up to order $\tilde{v}^{2}$. A similar expression holds for $\tilde{B}$. The effective theory for the gauge zero-modes is obtained by replacing these classical solutions back in the action Eq. (8). After expanding in derivatives to get an effective action which is local, we arrive at

$$
\begin{aligned}
S_{N}= & \int d^{4} x\left\{-\frac{1}{4} Z_{\mu \nu} Z^{\mu \nu}-\frac{1}{4} F_{\mu \nu} F^{\mu \nu}-\frac{1}{2} m_{Z}^{2} Z_{\mu} Z^{\mu}-e A_{\mu} J_{q}^{\mu}\right. \\
& \left.-\frac{e}{s c} Z_{\mu}\left[\left(c^{2}+\tilde{v}^{2} G_{f}^{3}\right) J_{3}^{\mu}-\left(s^{2}+\tilde{v}^{2} G_{f}^{B}\right) J_{Y}^{\mu}\right]-\frac{1}{2}\left[G_{f f}^{3} J_{\mu}^{3} J_{3}^{\mu}+G_{f f}^{B} J_{\mu}^{Y} J_{Y}^{\mu}\right]+\cdots\right\}
\end{aligned}
$$

where we neglected higher derivative terms, as well as terms of order $\tilde{v}^{4}$ and higher. In the above we defined the zero-mode fermion currents $J_{3}^{\mu}(x)=\bar{\psi}(x) \Gamma^{\mu} T^{3} \psi(x), J_{Y}^{\mu}(x)=\bar{\psi}(x) \Gamma^{\mu} Y \psi(x)$ and $J_{q}^{\mu}(x)=J_{3}^{\mu}(x)+J_{Y}^{\mu}(x)$, and used the shorthand notation

$$
\begin{aligned}
G_{f} & \equiv \int_{0}^{L} d y \tilde{G}_{0}(L, y)\left|f^{(0)}(y)\right|^{2} \\
G_{f f} & \equiv \int_{0}^{L} d y d y^{\prime}\left|f^{(0)}(y)\right|^{2} \tilde{G}_{0}\left(y, y^{\prime}\right)\left|f^{(0)}\left(y^{\prime}\right)\right|^{2}
\end{aligned}
$$


where $f^{(0)}(y)$ is the appropriate fermion zero-mode wavefunction (as defined in subsection 3.2). The propagators, $\tilde{G}_{0}\left(y, y^{\prime}\right)$, in Eqs. (22) are now given by Eq. (16), with the zero-mode part subtracted as in Eq. (14) and evaluated at zero momentum, $p=0$. The superscripts 3 or $B$ in the propagator terms appearing in Eq. (21) refer to the $W^{3}$ and $B$ respectively. We also wrote the action in the photon- $\mathrm{Z}$ basis

$$
\begin{aligned}
& A_{\mu}=s W_{\mu}^{(0)}+c B_{\mu}^{(0)} \\
& Z_{\mu}=c W_{\mu}^{(0)}-s B_{\mu}^{(0)},
\end{aligned}
$$

where $c=g / \sqrt{g^{2}+g^{\prime 2}}$ and $s=g^{\prime} / \sqrt{g^{2}+g^{\prime 2}}$. Finally, the $Z$ mass is given by

$$
m_{Z}^{2}=\frac{e^{2} \tilde{v}^{2}}{s^{2} c^{2}}\left\{1+\tilde{v}^{2}\left[\tilde{G}_{0}^{3}(L, L)+\tilde{G}_{0}^{B}(L, L)\right]+\mathcal{O}\left(v^{4}\right)\right\} .
$$

The second line in Eq. (21) contains the corrections to the $Z$-boson gauge couplings to fermions due to the deformation of the zero-mode wavefunctions induced by the Higgs VEV, and the four-fermion interactions induced by the electrically neutral KK towers. The first term in the second line of Eq. (21) can be rewritten as

$$
-\frac{e}{s_{*} c_{*}} Z_{z *}^{1 / 2}\left[J_{3}^{\mu}-s_{*}^{2} J_{q}^{\mu}\right]
$$

where

$$
s_{*}^{2}=s^{2}\left[1+\tilde{v}^{2}\left(\frac{c^{2}}{s^{2}} G_{f}^{B}-G_{f}^{3}\right)\right]
$$

and

$$
Z_{z *}=1+\frac{\tilde{v}^{2}}{s^{2} c^{2}}\left(c^{2} G_{f}^{B}+s^{2} G_{f}^{3}\right) .
$$

We shall use Eqs.(26) and Eq. (27) in computing precision electroweak parameters later on.

The charged gauge sector can be handled in a similar fashion and it is easy to see that the result of integrating out the massive states is

$$
\begin{aligned}
S_{C}= & \int d^{4} x\left\{-\frac{1}{2} W_{\mu \nu}^{+} W_{-}^{\mu \nu}-m_{W}^{2} W_{\mu}^{+} W_{-}^{\mu}\right. \\
& \left.-\frac{e}{\sqrt{2} s}\left(1+\tilde{v}^{2} G_{f}^{3}\right)\left[W_{\mu}^{+} J_{+}^{\mu}+W_{\mu}^{-} J_{-}^{\mu}\right]-\frac{1}{2} G_{f f}^{3} J_{\mu}^{+} J_{-}^{\mu}+\cdots\right\} .
\end{aligned}
$$

where

$$
m_{W}^{2}=\frac{e^{2} \tilde{v}^{2}}{s^{2}}\left\{1+\tilde{v}^{2} \tilde{G}_{0}^{3}(L, L)+\mathcal{O}\left(v^{4}\right)\right\} .
$$

and $J_{ \pm}^{\mu}(x)=\bar{\psi}(x) \Gamma^{\mu} T_{ \pm} \psi(x)$, with $T_{ \pm}=T^{1} \pm i T^{2}$, are the charged fermion currents. 


\subsection{Properties of Bulk Fermions}

We now consider the fermions in more detail. The properties of bulk fermions in an $\mathrm{AdS}_{5}$ background are well-known (see e.g. [18]), but we summarize the relevant results, both for the sake of completeness and to establish notation. For simplicity we shall ignore the effect of local kinetic terms for the fermion fields. In this case, the free action for a 5-dimensional fermion is given by

$$
S_{\Psi}=-\int_{0}^{L} d^{4} x d y \sqrt{-G}\left\{i \bar{\Psi} \Gamma^{A} e_{A}^{M} D_{M} \Psi+i M \bar{\Psi} \Psi\right\}
$$

where $\Gamma^{A}$ are the flat space 5-dimensional gamma matrices, $D_{M}$ is a covariant derivative, with respect to both gauge and general coordinate transformations, $G$ is the metric defined in Eq. (1D), $e_{A}{ }^{M}$ is the corresponding vielbein, and $M=c \sigma^{\prime}$ is an (odd) bulk mass term. Expanding the fermion field in Kaluza-Klein modes, $\Psi_{L, R}(x, y)=e^{3 \sigma / 2} \sum_{n} \psi_{L, R}^{(n)}(x) f_{L, R}^{n}(y)$, one finds that the KK mode wavefunctions so defined satisfy

$$
\left[\partial_{y}-\frac{1}{2} \sigma^{\prime} \pm M\right] f_{L, R}^{n}= \pm m_{n} e^{\sigma} f_{R, L}^{n}
$$

where the $+(-)$ sign applies to the wavefunction of left-handed (right-handed) 4-dimensional fermions [we take $P_{L, R}=\frac{1}{2}\left(1 \pm \Gamma^{5}\right)$ ]. The orthonormality conditions required to obtain canonically normalized kinetic terms in the effective 4-dimensional theory read

$$
\int_{0}^{L} d y f^{n}(y) f^{m}(y)=\delta_{n m} .
$$

Of special relevance are the zero-modes, which satisfy

$$
\left[\partial_{y}-\left(\frac{1}{2} \mp c\right) \sigma^{\prime}\right] f_{L, R}^{0}=0
$$

and are therefore given by

$$
f_{L, R}^{0}(y)=\sqrt{\frac{k(1 \mp 2 c)}{e^{(1 \mp 2 c) k L}-1}} e^{\left(\frac{1}{2} \mp c\right) \sigma} .
$$

Depending on the fermion $Z_{2}$ parity, one of these zero-modes is projected out and the remaining one has an exponential profile that is localized towards one of the orbifold fixed points (depending on whether $c>1 / 2$ or $c<1 / 2$ ). In order to simplify the discussion we will adopt a convention for the bulk mass term of a given fermion $f$ such that $c_{f}>1 / 2\left(c_{f}<1 / 2\right)$ corresponds to the physical zero mode being localized towards the UV brane (IR brane). For 
example, if $\Psi_{Q}$ and $\Psi_{u}$ denote the 5-dimensional fermion fields corresponding to the left-handed quark doublet and right-handed up quark $S U(2)$ singlet respectively, then we define $c_{Q}$ and $c_{u}$ by

$$
\begin{aligned}
S=-\int_{0}^{L} d^{4} x d y \sqrt{-G} & \left\{i \bar{\Psi}_{Q} \Gamma^{A} e_{A}{ }^{M} D_{M} \Psi_{Q}+i \bar{\Psi}_{u} \Gamma^{A} e_{A}{ }^{M} D_{M} \Psi_{u}\right. \\
& \left.+i c_{Q} \sigma^{\prime} \bar{\Psi}_{Q} \Psi_{Q}-i c_{u} \sigma^{\prime} \bar{\Psi}_{u} \Psi_{u}+\cdots\right\}
\end{aligned}
$$

Therefore, the zero-mode wavefunctions will always be given by

$$
f^{0}(y)=\sqrt{\frac{k\left(1-2 c_{f}\right)}{e^{\left(1-2 c_{f}\right) k L}-1}} e^{\left(\frac{1}{2}-c_{f}\right) \sigma} .
$$

We will also need the higher KK mode wavefunctions. With the above convention for the bulk mass term, the $Z_{2}$ even and odd solutions for a given 5-dimensional fermion field, denoted by $f_{+}^{n}(y)$ and $f_{-}^{n}(y)$ respectively, are

$$
f_{ \pm}^{n}(y)=A_{n} e^{\sigma}\left[J_{\left|c_{f} \pm \frac{1}{2}\right|}\left(\frac{m_{n}}{k} e^{\sigma}\right)+b\left(m_{n} / k\right) Y_{\left|c_{f} \pm \frac{1}{2}\right|}\left(\frac{m_{n}}{k} e^{\sigma}\right)\right]
$$

where

$$
b(x)=-\frac{J_{\left|c_{f}-\frac{1}{2}\right|}(x)}{Y_{\left|c_{f}-\frac{1}{2}\right|}(x)} .
$$

The KK masses are determined by the condition $b\left(m_{n} / k\right)=b\left(e^{k L} m_{n} / k\right)$ and the normalization constants $A_{n}$ are determined by Eq. (32).

The localized Higgs VEV, which is responsible for the zero-mode fermion masses, induces mixing between the previous zero-mode and the massive KK tower in a way which is analogous to the gauge sector described in the previous section. This mixing is proportional to both the bare 5-dimensional Yukawa coupling and to the values of the KK wavefunctions at the VEV position, $y=L$. The zero-mode wavefunctions at $y=L$ can be obtained directly from Eq. (36)). As for the massive KK modes, we find that, for the lowest lying ones and for all moderate $c_{f}$, the wavefunctions evaluated at the IR brane are given to a good approximation by

$$
f_{+}^{n}(L) \approx \pm \sqrt{2 k}
$$

These mixing effects can be important in the top sector. In fact, since the effective 4dimensional top Yukawa coupling is close to one, it is necessary to move the top zero-mode wavefunctions closer to the IR brane $\left(c_{f}<1 / 2\right)$, or otherwise one would obtain an exponential suppression of the effective 4-dimensional Yukawa coupling. This would require one to invoke a 
5-dimensional Yukawa coupling which is non-perturbative. Even for values of $c_{f}$ which result in perturbative Yukawa interactions, the strong mixing enhances the loop effects of the KK modes of the top, which can render the theory incompatible with electroweak precision measurements.

The Yukawa term that couples the even towers associated with the left- and right-handed top has the following structure

$$
\begin{aligned}
\sqrt{-G} \delta(L-y) \frac{\hat{\lambda}_{5}}{\Lambda} \sqrt{2} v \bar{\Psi}_{t_{L}} \Psi_{t_{R}} \approx \delta(y-L) m_{t}\left[\bar{\psi}_{t_{L}}^{(0)} \psi_{t_{R}}^{(0)}+\frac{\sqrt{2 k L}}{a_{t_{R}}} \sum_{n \neq 0} \bar{\psi}_{t_{L}}^{(0)} \psi_{t_{R}}^{(n)}\right. \\
\left.+\frac{\sqrt{2 k L}}{a_{t_{L}}} \sum_{n \neq 0} \bar{\psi}_{t_{L}}^{(n)} \psi_{t_{R}}^{(0)}+\frac{2 k L}{a_{t_{L}} a_{t_{R}}} \sum_{n, m \neq 0} \bar{\psi}_{t_{L}}^{(n)} \psi_{t_{R}}^{(m)}\right]
\end{aligned}
$$

where we wrote the 5-dimensional top Yukawa coupling in Eq. (7) in units of the cutoff scale $\Lambda$ and introduced the dimensionless coupling $\hat{\lambda}_{5}$. We also defined the parameters

$$
a_{f}=\sqrt{\frac{\left(1-2 c_{f}\right) k L}{e^{\left(1-2 c_{f}\right) k L}-1}} e^{\left(1 / 2-c_{f}\right) k L} \approx \begin{cases}\sqrt{\left(2 c_{f}-1\right) k L} e^{-\left(c_{f}-1 / 2\right) k L} & c_{f}-1 / 2 \gtrsim 1 / 2 k L \\ 1 & c_{f}=1 / 2 \\ \sqrt{\left(1-2 c_{f}\right) k L} & 1 / 2-c_{f} \gtrsim 1 / 2 k L\end{cases}
$$

and the zero-mode mass

$$
m_{t}=a_{t_{L}} a_{t_{R}} \frac{\hat{\lambda}_{5}}{\Lambda L} \sqrt{2} \tilde{v} .
$$

First let us see what is required to reproduce the observed top mass, i.e. $y_{t} \equiv a_{t_{L}} a_{t_{R}} \hat{\lambda}_{5} /(\Lambda L) \sim$ 1. The volume suppression factor $1 /(\Lambda L)$ indicates that we may want to make $\hat{\lambda}_{5}$ as large as possible. A similar situation arises in the gauge sector. Since the 4-dimensional (unified) gauge coupling $g_{4}^{2} \approx \hat{g}_{5}^{2} /(\Lambda L)$ is of order one, and $\Lambda L>k L \sim 30$, we are led to take the dimensionless constant $\hat{g}_{5}$ as large as possible. Thus, it is natural to assume that both these interactions become strong at the cutoff scale $\Lambda>k$ and we can use NDA to estimate the size of the dimensionless couplings $\hat{g}_{5}$ and $\hat{\lambda}_{5}$. In this strong coupling limit, we have 12 ]

$$
\hat{g}_{5} \sim l_{5}, \quad \hat{\lambda}_{5} \sim \frac{l_{5}}{\sqrt{l_{4}}},
$$

where $l_{5}=24 \pi^{3}$ and $l_{4}=16 \pi^{2}$ are the five- and 4-dimensional loop factors respectively. Requiring that $g_{4} \sim 1$, we find $\Lambda L \sim l_{5}$. Note that this gives $\Lambda / k \sim l_{5} /(k L) \sim 25$ which shows that the gauge interactions get strong at a scale where the theory looks 5-dimensional, and therefore justifies the use of the above 5-dimensional NDA analysis. 
The top Yukawa coupling is then

$$
y_{t} \sim \frac{a_{t_{L}} a_{t_{R}}}{\sqrt{l_{4}}}=\frac{a_{t_{L}} a_{t_{R}}}{4 \pi},
$$

which, according to Eq. (41), can easily be of order one if $c_{t_{L}}, c_{t_{R}}$ are slightly below $1 / 2$.

As an aside, we may ask whether the gravitational interactions also get strong at the scale $\Lambda$ defined above. This will be the case if the 5 -dimensional Planck scale, $M_{5}$, is related to $\Lambda$ by $M_{5}^{3} \sim \Lambda^{3} / l_{5}$. However, if we take $k \sim 10^{16} \mathrm{GeV}$ as suggested by gauge coupling unification and $M_{P}=2 \times 10^{18} \mathrm{GeV}$, we find

$$
\frac{M_{5}^{3}}{\Lambda^{3} / l_{5}} \approx \frac{k M_{P}^{2}}{\Lambda^{3} / l_{5}}=l_{5}\left(\frac{M_{P}}{k}\right)^{2}\left(\frac{k}{\Lambda}\right)^{3} \sim 10^{3} .
$$

Therefore, for the previous choice of parameters the gravitational interactions are still weak when the gauge (and top Yukawa) interactions get strong.

Now let us go back to the effects of the massive KK top towers and the interactions given in Eq. (40). Since the requirement that the top mass be reproduced led us to consider $c_{t_{L}}, c_{t_{R}}<1 / 2$, it follows from Eqs. (40) and (41) that the mixing effects are proportional

to $m_{t} \sqrt{2 k L} / a_{t_{L, R}} \approx m_{t} /\left(\frac{1}{2}-c_{t_{L, R}}\right)^{1 / 2}$. These couplings can be considerably large if $c_{f}$ is close to $1 / 2$ (but still $1 / 2-c_{f}>1 / 2 k L$ ), thus imposing some further constraints on the allowed region of parameter space. In fact, even if these factors are small enough that we can treat these effects in the mass insertion approximation, there can still be sizable contributions to the $T$ parameter due to loops of the top KK modes. We estimate these contributions in the next section.

\section{Precision Electroweak Analysis}

As described above, there are two main contributions affecting the precision electroweak observables in this theory. The first type are the Higgs localization effects that are associated with a deformation of the zero-mode weak gauge boson wavefunctions and masses. These effects are related to the functions $G_{f}$ and $\tilde{G}_{0}(L, L)$ introduced in the previous section. When all fermions have a common value of the bulk mass parameter $c_{f}$, these affect the couplings of the gauge bosons to quarks and leptons in a universal way. They are therefore associated with oblique corrections. Also in this class are loop-level corrections to the gauge boson self-energies due to the KK modes of bulk fields. The second type of contributions arise from exchange of the KK 
modes of the gauge bosons at tree-level, leading to non-oblique corrections proportional to the functions $G_{f f}^{3, B}$ introduced in the previous section. Since the Higgs localization effects are associated with oblique corrections, their impact on all experimental observables can be described in terms of parameters $S, T$ and $U$, defined in Ref. [19]. These parameters provide an excellent description of all precision electroweak data in the case that the non-oblique corrections, parametrized by $G_{f f}^{i}$, are much smaller than the oblique ones, namely $G_{f f}^{i} \ll G_{0}^{i}, G_{f}^{i}$.

Whenever the non-oblique corrections are non-negligible, the above procedure cannot be applied. However, there are certain cases in which effective parameters $S_{\text {eff }}, T_{\text {eff }}$ and $U_{\text {eff }}$ may be defined in order to describe only a subset of the experimental data, which, due to its precision, leads to the most stringent experimental tests on the theory. This is the case, for instance, whenever the heavy KK modes of the neutral gauge fields are sufficiently heavy, the only impact of non-oblique corrections on $m_{W}$ and the Z-pole observables comes indirectly from the Fermi constant $G_{F}$. Using $G_{F}$ as an input value to the precision electroweak data, the non-oblique corrections induced by the heavy KK modes data can be easily absorbed into the definition of new, effective parameters $T_{\text {eff }}$ and $U_{\text {eff }}$ [10]. The advantage of this procedure is that the functional dependence of $m_{W}$ and the $Z$-pole observables on the new effective parameters is the same as the functional dependence of these observables on $S, T$ and $U$ in the case in which only oblique corrections are present, and thus, one can use the fits of $S, T$, and $U$ derived from such observables to understand the physical picture and constraint the parameters of the model.

\subsection{Massive Kaluza-Klein effects}

We start by considering the effects of the heavy KK modes in the theory. First, due to the large top Yukawa coupling, the top KK modes can induce potentially large contributions to the $T$ parameter. In fact, we saw in Eq. (40) that the localized Higgs VEV induces large mixings between the top-quark left and right zero modes and their even KK towers. Furthermore, the heavy KK modes receive electroweak breaking contributions to their masses that are enhanced by factors of $2 k L{ }^{1}$ The largest contributions to $T$ are dominated by graphs with the heavy KK modes running in the loop. These may be estimated in the mass insertion approximation. The contribution induced by the mixing of the first KK modes of the left- and right-handed

\footnotetext{
${ }^{1}$ These factors arise from the KK mode wavefunctions at the IR brane, and are also responsible for the strong coupling of the KK modes to IR brane fields.
} 
top quark is of order

$$
\Delta T_{t_{L}^{1}, t_{R}^{1}} \sim\left(\frac{2 k L}{a_{t_{L}} a_{t_{R}}}\right)^{4}\left(\frac{m_{t}}{m_{t}^{(1)}}\right)^{2}\left[\frac{N_{c}}{16 \pi s^{2} c^{2}}\left(\frac{m_{t}}{m_{Z}}\right)^{2}\right],
$$

where $m_{t}^{(1)}$ is the mass of the heaviest of these two KK modes, $a_{t_{L}}$ and $a_{t_{R}}$ were defined in Eq. (41), $s$ is the sine of the weak mixing angle, and the term in square brackets is the SM top contribution, which is of order one. Note that, due to the brane localized Yukawa interactions and the consequent mixing among KK modes exhibited in Eq. (40), summing up the KK tower leads to a quadratic sensitivity on the cutoff $\tilde{\Lambda}=\Lambda e^{-k L}{ }^{2}{ }^{2}$ This quadratic sensitivity depends strongly on the assumption that the couplings of the heavy KK modes are well described by Eq. (40). For example, the presence of IR brane localized kinetic terms for the top and bottom quark fields can suppress the couplings between the Higgs and the higher top KK modes [20] and lead to a milder sensitivity to the cutoff scale. Since we expect that the contribution of the lightest KK mode remains approximately unaffected by these effects, Eq. (46) provides a lower bound on the total effect of integrating out the heavy KK modes.

An upper bound on the order of magnitude of the contribution of the KK tower on $T$ may be obtained by a naive extrapolation of the sum over all KK modes up to the cutoff scale, which at the end of subsection 3.2 was estimated as $\tilde{\Lambda} \lesssim 25 k e^{-k L}$. For $c_{t_{L}}, c_{t_{R}}<1 / 2$ it reads $\Delta T \lesssim 60\left(\frac{1}{2}-c_{t_{L}}\right)^{-2}\left(\frac{1}{2}-c_{t_{R}}\right)^{-2}\left(m_{t} / m_{t}^{(1)}\right)^{2}$. Therefore, this contribution may be strongly suppressed for values of $c_{f} \ll 1 / 2$.

However, as shown in Ref. [10], when the fermions are localized towards the IR brane, $c_{f} \ll 1 / 2$, there are important non-oblique corrections and, in the absence of local gauge kinetic terms the bounds on the KK scale become tight [10. One possibility is to take the bulk mass parameters of the first and second generation larger than the one associated with the third generation. However, choosing different bulk masses for the fermions can potentially lead to large FCNC effects [17]. In particular, taking the third generation $c_{Q}$ very different from the first two generation $c_{f}$ 's can lead to dangerous contributions to $Z \rightarrow b \bar{b}$ [14]. Thus, in order to keep non-oblique corrections small, while suppressing the dangerous contributions to the $Z \rightarrow b \bar{b}$ decay width and flavor changing neutral currents we are led to take equal $c_{f}$ 's for the left-handed top and the first two generations of fermions. The constraints on $t_{R}$ are much weaker and it is possible to render the above contributions to $T$ negligible by taking $c_{t_{R}}$ sufficiently negative.

\footnotetext{
${ }^{2}$ This is similar to the model of Ref. [16], which contained large, uncalculabe, contributions to the $\rho$ parameter. Note, however, that in our case the compactification scale is about an order of magnitude higher.
} 
There are also contributions that are sensitive only to $c_{t_{L}}$, which are induced by the $\psi_{t_{L}}^{n}-\psi_{t_{R}}^{0}$ mixing. The effect of the first KK level is given by

$$
\Delta T_{t_{L}^{1}, t_{R}^{0}} \sim \frac{(2 k L)^{2}}{3 a_{t_{L}}^{4}}\left(\frac{m_{t}}{m_{t_{L}}^{(1)}}\right)^{2}\left[\frac{N_{c}}{16 \pi s^{2} c^{2}}\left(\frac{m_{t}}{m_{Z}}\right)^{2}\right]
$$

and, as before, one can estimate the KK contribution to $T$, which scales like $\log \tilde{\Lambda}$, by summing up the effects of the KK modes lighter than $\tilde{\Lambda}$. For $\Lambda \sim 25 k$, these type of contributions give $\Delta T_{K K} \lesssim 4\left(\frac{1}{2}-c_{t_{L}}\right)^{-2}\left(m_{t} / m_{t_{L}}^{(1)}\right)^{2}$.

The KK tower effects computed above can be considered as a conservative estimate of the total contribution to the $T$ parameter. Such effects can be rendered small by taking appropriate values of $c_{t_{L}}$ and $c_{t_{R}}$. For instance, considering $c_{t_{L}} \simeq 0.3$ and $c_{t_{R}} \simeq-5$, and using $m_{t_{L}}^{(1)} \gtrsim 2.5 k e^{-k L}$, these contributions are smaller than about $1 / 10$ of the dominant contribution induced by the oblique corrections to the gauge boson masses and wave functions, that will be discussed in more detail in the next section. Therefore, for $c_{f}=c_{t_{L}} \simeq 0.3, c_{t_{R}} \simeq-5$, where $c_{f}$ stands for the rest of the fermions, the above corrections have a negligible impact on the fit to the experimental data.

Let us remark that the effects of the physics above $\tilde{\Lambda}$ can be parametrized in the effective theory by local operators such as

$$
\mathcal{O}_{T}=\delta(y-L) \frac{c}{\Lambda^{2}}\left(H^{\dagger} D_{\mu} H\right)^{2} .
$$

Even when the unknown coefficient attains its strong coupling value, $c \sim 16 \pi^{2}$, their contribution to $T$ is negligible for $\Lambda \sim 25 k$ and $k e^{-k L}$ above a few $\mathrm{TeV}$.

There are possible additional effects induced by the presence of the GUT theory. The KK modes of the heavy $X$ and $Y$ bosons of the theory are essential for the question of unification of couplings, but can also induce important contributions to the precision electroweak observables. This is due to the fact that these gauge bosons form a doublet under $S U(2)$ and, if coupled to the Higgs, can lead to large contributions to the $T$ parameter, because the splitting in masses squared will be of order $\tilde{v}^{2}$, while their masses are of order TeV. In fact, if these gauge bosons are even about the IR brane, so that the IR brane respects the GUT symmetry, they give a contribution to $T$ of order

$$
\Delta T_{X Y} \sim \frac{N_{c}(2 k L)^{2}}{4 \pi s^{2}}\left(\frac{\tilde{v}}{m_{X Y}^{(1)}}\right)^{2},
$$

which is of order one when $m_{X Y}^{(1)}$, the mass of the first KK mode of the gauge bosons $X$ and $Y$ is of order of a few TeV. A straightforward way of cancelling these effects is by demanding the 
$X$ and $Y$ gauge bosons to be odd under the $Z_{2}$ orbifold associated with the IR brane. In this case, their couplings to the Higgs vanish and these additional contributions to the electroweak precision observables become negligible, because the $X Y$ masses are highly degenerate.

\subsection{Effective S, T and U parameters}

Having established that the possible effects from the heavy KK modes of the fermions and the GUT sector of the theory can be made negligible, we turn to the effects associated with the zero-mode sector and the non-oblique contributions to $G_{F}$. From the expression of the effective action for the charged gauge currents and $m_{W}$, Eqs. (28) and (29), respectively, one finds that $G_{F}$ is given by

$$
4 \sqrt{2} G_{F}=\frac{1}{\tilde{v}^{2}}\left[1+\tilde{v}^{2}\left(2 G_{f}^{3}-\tilde{G}_{0}^{3}(L, L)-G_{f f}^{3}\right)\right] .
$$

The last term in the above equation represents the non-oblique corrections. Observe that, with this normalization $\tilde{v} \simeq 123 \mathrm{GeV}$.

In the case the non-oblique corrections, proportional to the four-fermion interactions $G_{f f}$ are negligible, the corrections to all experimental data at the Z-pole, can be taken into account via the standard $S, T$ and $U$ parametrization. Following the procedure outlined in Ref. [19] and using Eqs. (26), (27) and (29), one find the values

$$
\begin{aligned}
\alpha S & \approx 4 \tilde{v}^{2}\left[s^{2} G_{f}^{3}+c^{2} G_{f}^{B}\right]+\mathcal{O}\left(\tilde{v}^{4}\right) \\
\alpha T & \approx \tilde{v}^{2}\left[2 G_{f}^{B}-\tilde{G}_{0}^{B}(L, L)\right]+\mathcal{O}\left(\tilde{v}^{4}\right) \\
\alpha U & \approx \mathcal{O}\left(\tilde{v}^{4}\right)
\end{aligned}
$$

As mentioned above, in many cases the only relevant non-oblique corrections to $m_{W}$ and the Z-pole observables come indirectly through the Fermi constant $G_{F}$. In this case, following Ref. [10], and considering the expression of $G_{F}$, Eq. (150), it is possible to define the effective parameters $S_{\text {eff }}, T_{\text {eff }}$ and $U_{\text {eff }}$, which are given by,

$$
\begin{aligned}
\alpha S_{\mathrm{eff}} & \approx 4 \tilde{v}^{2}\left[s^{2} G_{f}^{3}+c^{2} G_{f}^{B}\right]+\mathcal{O}\left(\tilde{v}^{4}\right) \\
\alpha T_{\mathrm{eff}} & \approx \tilde{v}^{2}\left[2 G_{f}^{B}-\tilde{G}_{0}^{B}(L, L)+G_{f f}^{3}\right]+\mathcal{O}\left(\tilde{v}^{4}\right) \\
\alpha U_{\mathrm{eff}} & \approx-4 s^{2} \tilde{v}^{2} G_{f f}^{3}+\mathcal{O}\left(\tilde{v}^{4}\right)
\end{aligned}
$$

For completeness, we shall also give the expression for $\rho_{*}(0)$, which is defined as the lowenergy ratio of neutral to charged current interactions. These low-energy observables depend 
on the following combinations

$$
\Delta^{3, B}=2 G_{f}^{3, B}-G_{f f}^{3, B}-\tilde{G}_{0}^{3, B}(L, L)
$$

In terms of $\Delta^{3, B}$, the effective charged four-fermion interaction, which determines the Fermi constant, $G_{F}$, from muon decay, is given by

$$
\mathrm{CC}=\frac{1}{\tilde{v}^{2}}\left\{1+\tilde{v}^{2} \Delta^{3}+\mathcal{O}\left(\tilde{v}^{4}\right)\right\} \equiv G_{F}
$$

Similarly, the neutral four-fermion interaction that is relevant in neutrino-nucleon scattering can be written as

$$
\mathrm{NC}=T_{\nu}^{3}\left(T_{q}^{3}-\bar{s}^{2} Q_{q}\right) \frac{1}{\tilde{v}^{2}}\left\{1+\tilde{v}^{2}\left[\Delta^{3}+\Delta^{B}\right]+\mathcal{O}\left(\tilde{v}^{4}\right)\right\}
$$

where

$$
\bar{s}^{2}=s^{2}\left\{1+\tilde{v}^{2}\left[G_{f f}^{3}-G_{f}^{3}-\frac{c^{2}}{s^{2}}\left(G_{f f}^{B}-G_{f}^{B}\right)\right]+\mathcal{O}\left(\tilde{v}^{4}\right)\right\} .
$$

It follows that

$$
\rho_{*}(0)=1+\tilde{v}^{2} \Delta^{B}+\mathcal{O}\left(\tilde{v}^{4}\right)
$$

It is important to stress that, in the case in which all SM fermions are localized on the infrared brane, $G_{f f}^{i}=G_{f}^{i}=G_{0}^{i}(L, L)$ and therefore $\Delta^{3, B}=0$. Consequently, in this case $\rho_{*}(0)=1$, while $\bar{s}^{2}=s^{2}$.

\subsection{Explicit Expressions for $G_{f}, G_{f f}$ and $\tilde{G}_{0}(L, L)$}

We have established that the most important new effects in the low-energy theory are contained in $G_{f}, G_{f f}$ and $\tilde{G}_{0}(L, L)$, which we now evaluate in terms of the fundamental parameters of the 5-dimensional theory. For simplicity, in this subsection we assume that we have a simple gauge group. The application to the $S U(2) \times U(1)$ theory is straightforward: one simply plugs the relevant expressions for the $S U(2)$ and $U(1)$ sectors into the formulae of the previous section. As we have seen in Eqs. (24) and (29), the corrections to the gauge boson masses are determined by $\tilde{G}_{0}(L, L)$, which is given by

$$
\tilde{G}_{0}(L, L)=-\frac{e^{2 k L} g^{2}}{k^{2}} \frac{2 k^{2}\left(L+r_{U V}\right)^{2}-2 k\left(L+r_{U V}\right)+1}{4 k\left(L+r_{U V}+r_{I R}\right)} .
$$

The corrections to the fermion-gauge boson vertices and the effects of the massive KK modes are contained in $G_{f}$ and $G_{f f}$. These depend on the fermion zero-mode wavefunctions 
and therefore on the parameter $c_{f}$, which we are taking to be common for all the fermions with the exception of $c_{t_{R}}$, as discussed in subsection 3.2. Using the zero-mode wavefunctions given in Eq. (36) to evaluate Eqs. (22), their exact analytic expressions can be obtained in a straightforward manner, but unfortunately the results have a somewhat complicated dependence on $c_{f}$. However, there are some limits in which the expressions simplify considerably. There are three qualitatively different cases depending on whether $c_{f}-\frac{1}{2}>1 / 2 k L, \frac{1}{2}-c_{f}>1 / 2 k L$ or $c_{f} \approx \frac{1}{2}$. These cases correspond to whether the fermion zero-mode wavefunction is localized towards the UV brane $\left(c_{f}>\frac{1}{2}\right)$, towards the IR brane $\left(c_{f}<\frac{1}{2}\right)$ or the conformal case $c_{f}=\frac{1}{2}$.

- $c_{f}-\frac{1}{2}>1 / 2 k L$ : The zero-mode fermions are localized towards the UV brane. Neglecting exponentially small terms we find that,

$$
\begin{aligned}
G_{f} & =\frac{e^{2 k L} g^{2}}{k^{2}} \frac{k\left(L+r_{U V}\right)-1-k r_{I R}+2 k^{2} r_{I R}\left(L+r_{U V}\right)}{4 k\left(L+r_{U V}+r_{I R}\right)} \\
G_{f f} & =-\frac{e^{2 k L} g^{2}}{k^{2}} \frac{2 k^{2} r_{I R}^{2}+2 k r_{I R}+1}{4 k\left(L+r_{U V}+r_{I R}\right)}
\end{aligned}
$$

where $g$ is the (zero-th order) zero-mode gauge coupling defined in Eq. (13) and we have dropped exponentially suppressed terms of order $e^{-k L}$. The results in Eqs. (59) and (60) are independent of $c_{f}$.

From these expressions we also find that the $\Delta$ parameter, Eq. (153), that enters in the low-energy observables is given by

$$
\Delta=2 G_{f}-\tilde{G}_{0}(L, L)-G_{f f}=\frac{e^{2 k L} g^{2}}{2 k^{2}} k\left(L+r_{U V}+r_{I R}\right) .
$$

- $c_{f}=\frac{1}{2}$ : This is the conformal case in which the zero-mode fermions couple with equal strength at all points along the extra dimension. We find

$$
\begin{aligned}
G_{f} & =\frac{e^{2 k L} g^{2}}{k} \frac{r_{U V}\left(1+2 k^{2} L r_{I R}\right)-k r_{U V}\left(L+r_{U V}+r_{I R}\right)+r_{I R}\left(2 k^{2} L^{2}-2 k L+1\right)}{4 k^{2} L\left(L+r_{U V}+r_{I R}\right)} \\
G_{f f} & =-e^{2 k L} g^{2} \frac{r_{U V}^{2}-2(k L-1) r_{U V} r_{I R}+\left(2 k^{2} L^{2}-2 k L+1\right) r_{I R}^{2}}{4 k^{3} L^{2}\left(L+r_{U V}+r_{I R}\right)}
\end{aligned}
$$

and

$$
\Delta=\frac{e^{2 k L} g^{2}}{2 k^{2}}\left(\frac{2 k^{2} L^{2}-2 k L+1}{2 k^{2} L^{2}}\right) k\left(L+r_{U V}+r_{I R}\right) .
$$

Note that Eqs. (62) and (63) vanish when $r_{U V}=r_{I R}=0$. This is a consequence of the gauge orthogonality condition, Eq. (12), and the fact that in the conformal case the fermion zero-mode 
wavefunction is flat and therefore proportional to the gauge zero mode wavefunction. Thus, the coupling of the zero-mode fermions to the higher KK gauge modes vanishes identically in this case.

- $\frac{1}{2}-c_{f}>1 / 2 k L$ : When the fermions are localized towards the IR brane the expressions for $G_{f}$ and $G_{f f}$ have a very complicated dependence on $c_{f}$ and we do not present them here. However, the linear combination corresponding to $\Delta$ simplifies to

$$
\Delta=\frac{e^{2 k L} g^{2}}{4\left(1-c_{f}\right) k^{2}} k\left(L+r_{U V}+r_{I R}\right)
$$

Finally, we emphasize again that in the limit where the fermions are localized on the IR brane, we have

$$
G_{f}=G_{f f}=\tilde{G}_{0}(L, L)
$$

where $\tilde{G}_{0}(L, L)$ was given in Eq. (58). These expressions can also be formally obtained by taking the limit $c_{f} \rightarrow-\infty$.

Note that, after multiplying by $\tilde{v}^{2}$, all the corrections are of order $g^{2} v^{2} / k^{2}$ times a function of $k L, k r_{U V}$ and $k r_{I R}$, which may represent an enhancement or a suppression depending on the location of the fermions. The corrections to the gauge boson masses, determined by $\tilde{G}_{0}(L, L)$, are always enhanced by a factor of order $k L$. When the fermions are localized towards the UV brane, we have $G_{f f} \ll G_{f} \ll \tilde{G}_{0}(L, L)$. The conformal case is somewhat special, since $G_{f}$ and $G_{f f}$ vanish when $r_{I R}=r_{U V}=0$. For the values of the brane kinetic coefficients that interest us ${ }^{3}, k r_{U V}^{\prime} \simeq 10$ and $k r_{U V} \simeq-10$, it is still true that $\tilde{G}_{0}(L, L)$ dominates over $G_{f}$ and $G_{f f}$. In the limit that the fermions are localized towards the IR brane, one generically has $G_{f f} \lesssim G_{f} \lesssim \tilde{G}_{0}(L, L)$. Note also that unless $c_{f}$ is very large and negative, $\Delta$ has always the same order of magnitude, independently of the location of the fermions, and is enhanced by $\mathcal{O}(k L)$. The behavior of $G_{f}$ and $G_{f f}$ as a function of $c_{f}$ can be seen in Fig. 1. In Fig. 2, we show the relative size of $G_{f}$ and $G_{f f}$ compared to $\tilde{G}_{0}(L, L)$ for $0<c_{f}<1$. We observe that the non-oblique corrections coming from $G_{f f}$ are much smaller than the oblique contributions $G_{f}$ and $\tilde{G}_{0}(L, L)$ in this region.

\footnotetext{
${ }^{3}$ The values of the local brane couplings $r_{U V}$ have been estimated by assuming, as in Eq. (3), that they include all the large logarithmic contributions, that lead to the difference between the high- and low-energy gauge couplings
} 

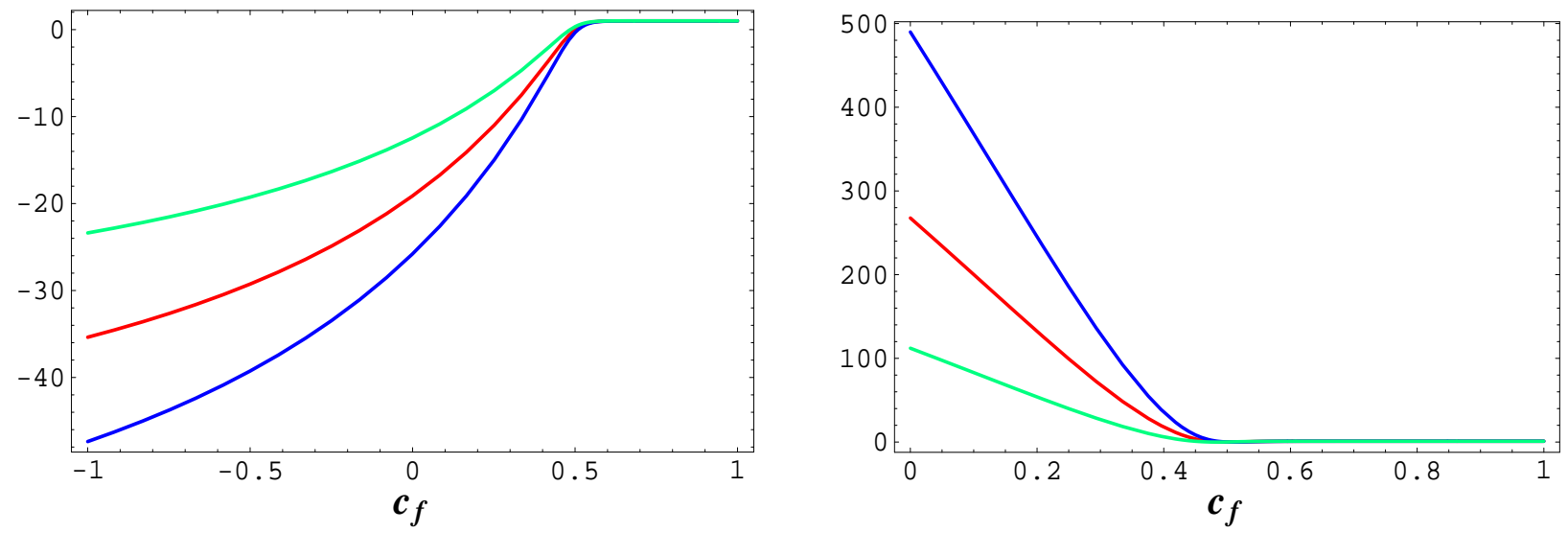

Figure 1: Behavior of $G_{f} / G_{f}^{\infty}$ (left) and $G_{f f} / G_{f f}^{\infty}$ (right) as a function of $c_{f}$, where the superscript $\infty$ indicates $c_{f}=+\infty$ (fermions localized on the UV brane). The curves correspond to $k r_{U V}=0$ (red), $k r_{U V}=10$ (blue) and $k r_{U V}=-10$ (green). In all of them we took $k r_{I R}=0$ and $k L=30$.

\section{$5 \quad$ Precision Electroweak Measurement Constraints}

In this section, we present the analysis of the precision electroweak data. As we have discussed in the previous section, whenever the effect of the four-fermion interactions $G_{f f}$ becomes negligible, the oblique parameters $S, T$ and $U$, Eq. (51) lead to a good parametrization of the corrections to all relevant experimental data. In this case, the effective parameter $U$ is equal to zero within the order of approximation we are using and therefore one can effectively reduce the fit to the experimental data to a two parameter fit. We shall consider as input parameters the experimental values of the mass $M_{Z}$, the Fermi constant $G_{F}$ and the electromagnetic gauge coupling. This will determine some of the underlying parameters of the model. Based on the grand unified RS scenario we take $g_{5}=\sqrt{5 / 3} g_{5}^{\prime}$. From Eq. (3) , taking $k \sim M_{G U T} \sim 10^{16} \mathrm{GeV}$, we estimate that the size of the log term in $r_{U V}^{\prime}$ should be roughly $L / 3$ provided the bare $r_{U V}^{\prime}$ is small and $b_{1}$ not much different from its SM value. Our results are rather insensitive to this choice. From here, for each given value of $L$ and $r_{I R}$, we fix $\tilde{v}, g_{5}$ and $r_{U V}$ using the experimental values of $G_{F}, \alpha$ and $M_{Z}$ (c.f. Eq. (50), Eq. (24) and Eqs. (13)). We will vary the remaining free parameters, $r_{I R}, L$, and the Higgs mass, to see which regions of parameters are in accord with precision measurements.

An inspection of the results presented in section 4.3 leads to the conclusion that, whenever $c_{f} \gtrsim 0.3$, the four-fermion interactions may be safely neglected. On the other hand, values of $c_{t_{L}}$ and $c_{t_{R}}$ smaller than about 0.5 may be needed in order to avoid large contributions to the $T$ parameter induced by KK fermion loops. In order to minimize the contribution of the KK 

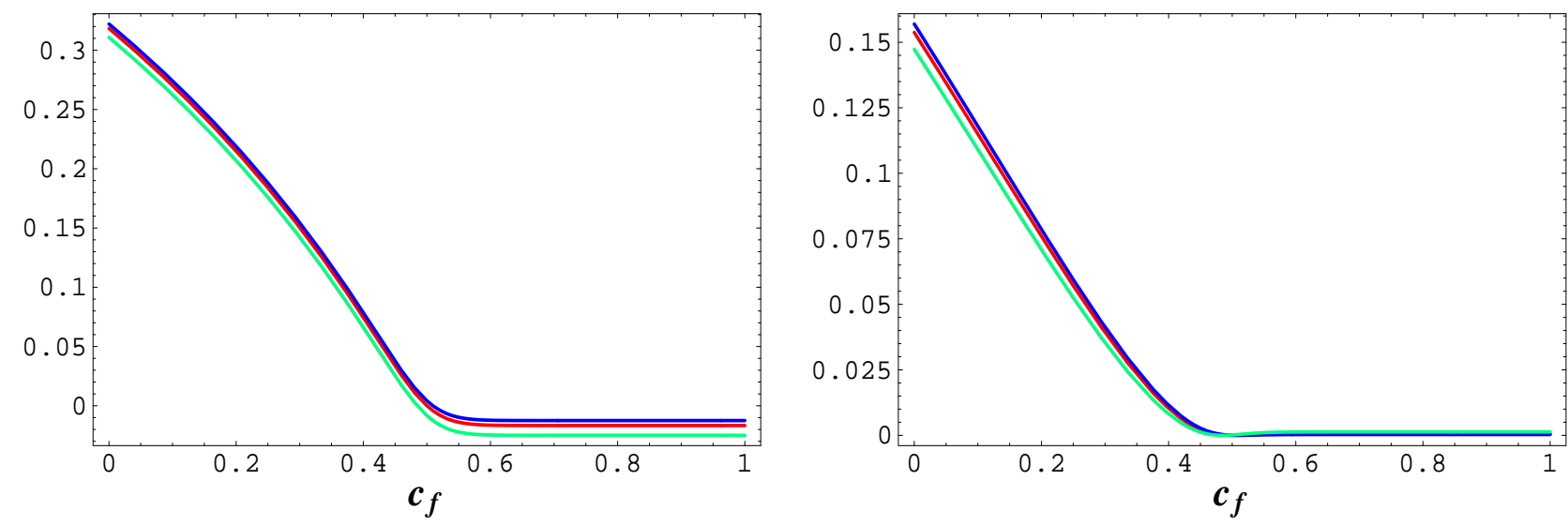

Figure 2: Behavior of $G_{f} / \tilde{G}_{0}(L, L)$ (left) and $G_{f f} / \tilde{G}_{0}(L, L)$ (right) as a function of $c_{f}$. The curves correspond to $k r_{U V}=0$ (red), $k r_{U V}=10$ (blue) and $k r_{U V}=-10$ (green). In all of them we took $k r_{I R}=0$ and $k L=30$.

fermion modes to the $T$ parameter, one can proceed with the localization of the third family in the infrared brane, as suggested in Ref. 14. Alternatively, choosing the value of $c_{t_{R}}<-5$, while keeping all other fermion bulk mass parameters $c_{f} \lesssim 0.5$, is sufficient to suppress the most important loop-induced contributions to the precision electroweak parameters.

In this work, we shall choose $c_{t_{R}} \simeq-5$, while for all other fermions $c_{f}=0.3$. This procedure keeps all light fermions at the same location in the bulk. This choice of bulk mass parameters $c_{f}$ has the advantage, compared to the case in which all the third generation fermions are localized on the IR brane, that flavor changing neutral current contributions are suppressed, with the largest FCNC effects in the right-handed up-type sector, inducing a $Z$ - $t_{R^{-}} c_{R}$ interaction ${ }^{4}$. Furthermore, large additional contributions to $R_{b}$ arising from the mismatch of the $Z$ coupling to bottom quarks compared to other fermions are avoided. Finally, for these values of the parameters, the description of the $Z$-pole observables and $m_{W}$ in terms of the oblique parameters $S, T$ and $U$ remains valid, and should be computed by universally taking $c_{f}=0.3$. For $c_{f}=0.3$ the value of $\tilde{G}_{0}(L, L)$ is much larger than $G_{f}$ and $G_{f f}$. From Eq. (51), and the results presented in Figure 2, one immediately observes that the theory tends to induce relatively large values of the $T$ parameter and small contributions to the parameter $S$.

These extra dimensional contributions to $S$ and $T$ must be combined with the more standard contributions from the Higgs. Compared to some reference Higgs mass $m_{r e f}^{2}$ (which must be

\footnotetext{
${ }^{4}$ The size of this interaction can be estimated assuming that the right-handed rotation matrices are of the order of the corresponding CKM elements, resulting in a $Z-t-c$ coupling strength of $10^{-6} \mathrm{~g}$. This is well below existing low energy bounds and is too small to affect single top production at the LHC [21].
} 
specified when performing the fit), the Higgs contributions are [19],

$$
\begin{aligned}
S_{H} & \simeq \frac{1}{12 \pi} \log \left(\frac{m_{h}^{2}}{m_{r e f}^{2}}\right) \\
T_{H} & \simeq-\frac{3}{16 \pi c_{0}^{2}} \log \left(\frac{m_{h}^{2}}{m_{r e f}^{2}}\right) \\
U_{H} & \simeq 0 .
\end{aligned}
$$

Thus, we observe that the large positive corrections to the $T$ parameter from the extra dimensions may be cancelled by the large negative corrections associated with a massive Higgs boson in the standard 'conspiracy' scenario familliar when there are weak scale vector-like quarks which mix with top [22]. Therefore, it is natural to expect that in this case the bounds on the KK gauge boson masses may be relaxed by taking large values of $m_{H}$. On the other hand, a large Higgs boson mass also induces positive corrections to the parameter $S$. However, for $c_{f} \simeq 0.3$, this contribution is also cancelled by the one coming from KK modes, which turns out to be negative and comparable to the one coming from the Higgs, for approximately the same values of the KK masses as the ones needed to cancel the $T$ contribution.

\subsection{Numerical Results}

In Ref. [10] we demonstrated that, in the case of light KK modes, the fit to the precision electroweak data is greatly improved by the presence of brane gauge kinetic terms. The main reason for this improvement is associated with the fact that for the same values of $k$ and $L$, the mass of the first gauge boson KK mode becomes significantly lower for large values of $r_{I R}^{i}$, than in the case of vanishing brane kinetic terms. However, since we are interested in the question of unification of couplings, we cannot set $r_{I R}^{i}$ to arbitrarily large values. Of course, if the $r_{I R}^{i}$ are unified, they will not disrupt the unification of the gauge couplings. However, since we must impose orbifold breaking of the GUT symmetry on the IR brane to suppress the contributions from loops of the broken gauge bosons to $T$, there is no reason to expect that the various $r_{I R}^{i}$ would be unified, and thus the prediction of unified gauge couplings could be lost. Therefore, we shall only concentrate on small values of $k r_{I R}^{i} \lesssim 2$, assuming that they are the same for both electroweak gauge fields, and thus do not disrupt unification. Note that even should they not be unified, $k r_{I R}$ of order 2 will change the unification conditions by roughly the same order of magnitude as the threshold corrections needed for RS unification anyway. Thus, our results may be taken as indicitive of the picture for small but non-unified IR brane terms as well. 


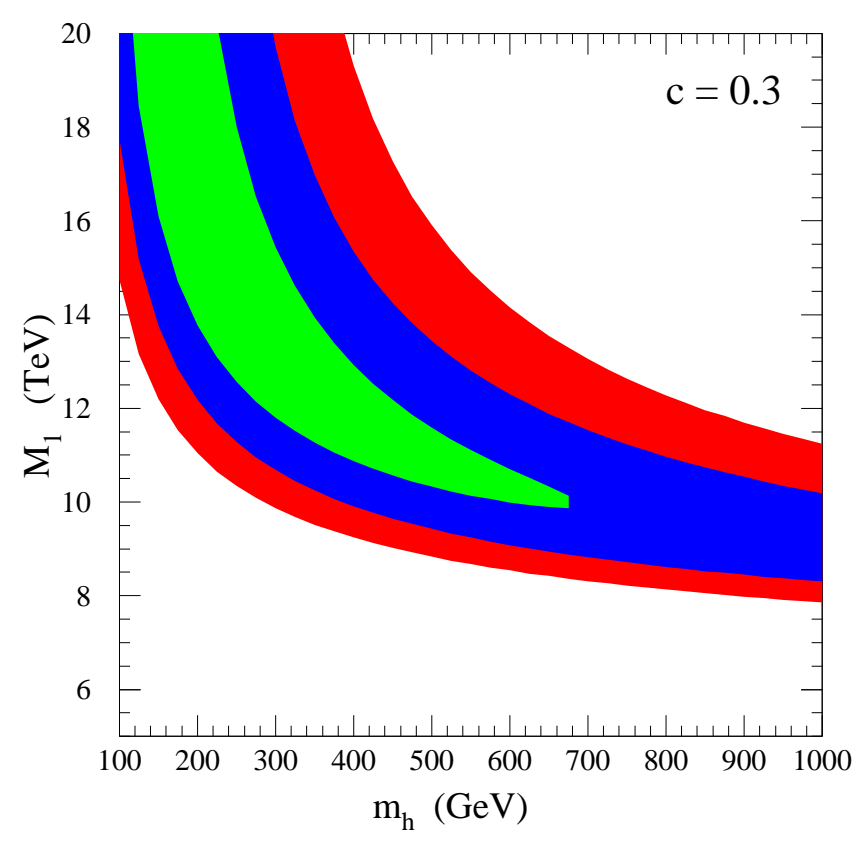

Figure 3: Allowed bands of the RS unified model in the parameter space of the Higgs mass and the first KK mode mass. The central green band represents $1 \sigma$ agreement with the electroweak fit to $S$ and $T$ (with $U=0$ ), whereas the surrounding blue and red bands indicate $2 \sigma$ and $3 \sigma$ agreement, respectively.

Our framework has $U \simeq 0$, and thus we choose to compare the RS model with the LEP electroweak working group fit to $S$ and $T$ which imposes $U=0$ in the fit [23]. In Figures 314 we present the results for the upper bound on the first weak gauge boson KK mass $M_{K K}$ as a function of the Higgs mass for $c_{f}=0.3$ (for all fermions except the top right, as explained above), and for (small) different values of $r_{I R}$. As anticipated, the fit to the data is significantly improved by large values of the Higgs mass $m_{h} \gtrsim 300 \mathrm{GeV}^{5}$. However, the bound on the first weak gauge boson KK mode mass is still about $11 \mathrm{TeV}$ and therefore difficult to detect at the LHC. However, even the addition of small kinetic terms in the infrared brane may have dramatic effects in the spectrum. Indeed, in the case of $k r_{I R}^{\prime}=k r_{I R}^{2}=2\left(k r_{I R}^{\prime}=k r_{I R}^{2}=1\right)$ a bound of about $4 \mathrm{TeV}(5 \mathrm{TeV})$ may be obtained for values of the Higgs mass larger than $400 \mathrm{GeV}$. Even in the case of a light Higgs boson, $m_{h}<200 \mathrm{GeV}$, one can accomodate values

\footnotetext{
${ }^{5}$ Note that while such large Higgs masses are generally incompatible with unification because the Higgs self-interaction typically reaches a Landau pole before the GUT scale, in the RS scenario fields localized on the IR brane see an effective cut-off of order $\tilde{\Lambda}=\Lambda e^{-k L}$, relaxing this constraint.
} 

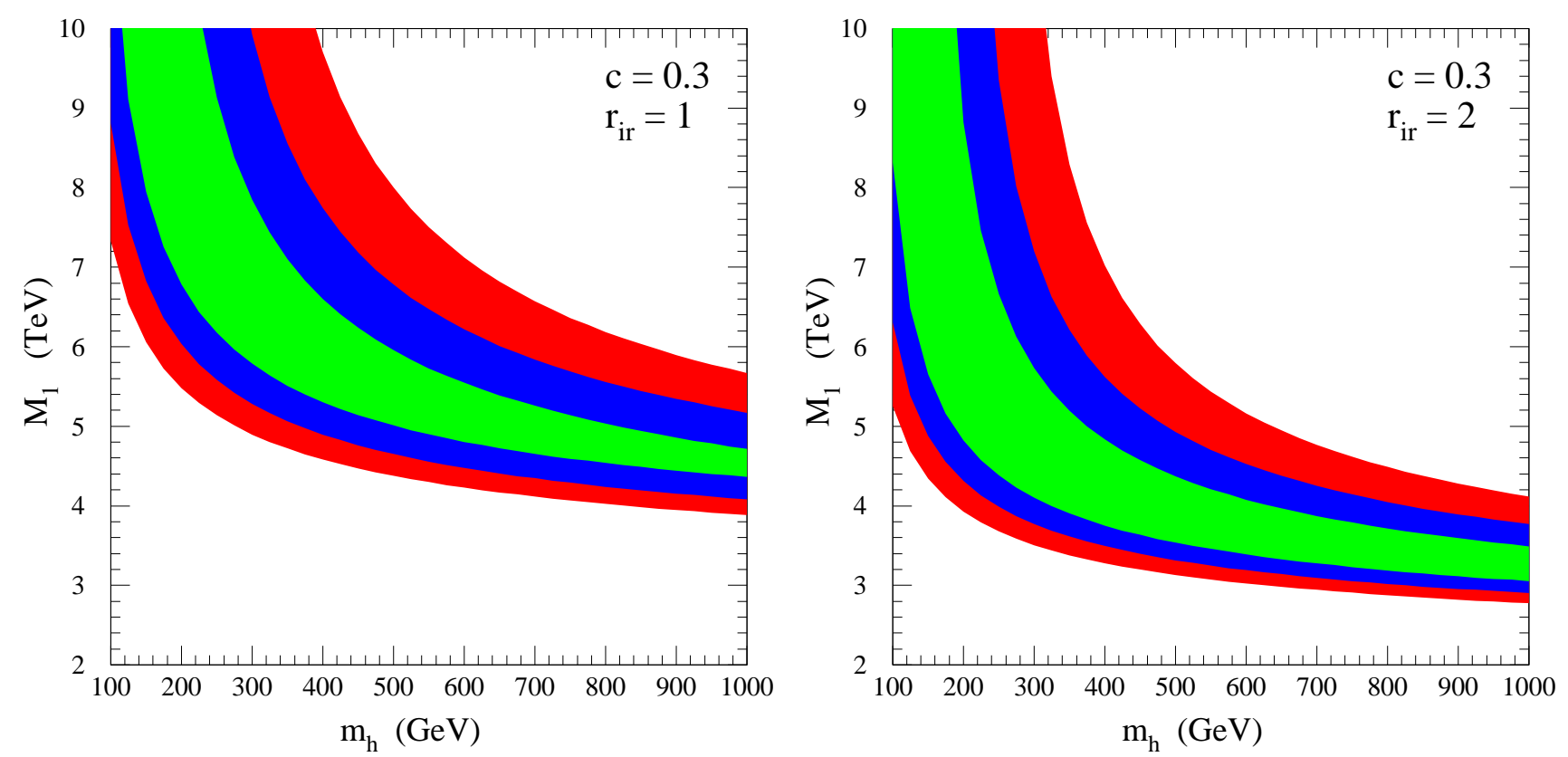

Figure 4: As Fig. 3, but with non-zero (unified) kinetic terms on the IR brane of $k r_{I R}=1,2$.

of the gauge boson $\mathrm{KK}$ masses of about $5 \mathrm{TeV}$ in a way consistent with precision electroweak data. Observe that even for large values of the Higgs mass, of about $1 \mathrm{TeV}$, a good fit to the precision electroweak data can be obtained, due to the simultaneous cancellation of the $T$ and $S$ contributions coming from the Higgs and extra dimensional effects discussed at the end of the last section.

Given the above conclusions, it seems possible that the LHC can study the prospect of RS unification. The electroweak data requires the masses of the first KK gauge bosons to be around $11 \mathrm{TeV}$ (a few TeV if the $k r_{I R}$ are allowed to be as large as 2 and the Higgs mass $\gtrsim 300 \mathrm{GeV}$ ). Given that this over-all scale is large compared to $\tilde{v}$, one thus expects quasi-degenerate $S U(3)$, $S U(2), U(1)$, and $X Y$ gauge bosons. Even at $8 \mathrm{TeV}$, it may be possible to see signs of the KK gluons indirectly, e.g. as $\bar{q} q \bar{q} q$ operators one might search for as a sign of quark compositeness. For masses in the range of a few $\mathrm{TeV}$, there is the hope that the GUT sector could be produced and studied, providing clear experimental evidence of an RS unified theory. 


\section{Conclusions}

Warped extra dimensional scenarios have the remarkable property of leading to a logarithmic dependence on the fundamental scale $k$ in the difference between the low energy couplings of the model. Since the logarithmic dependence is controlled by the 4-dimensional beta functions of the theory, this implies that the question of unification may be studied at a similar level of precision as in the four dimensional case.

In this work we studied a particular realization of these scenarios that is consistent with unification of couplings, and leads to the SM as the low energy effective theory. While the Higgs field is localized in the IR brane, in a way consistent with the Randall-Sundrum solution to the hierarchy problem, the gauge bosons and the fermions fields propagate in the bulk.

We introduce a formalism that allows to study the main corrections to the precision electroweak data in warped extra dimensions in the case in which the gauge fields propagate in the bulk. These corrections are parametrized by three set of functions $G_{f f}, G_{f}$ and $G_{0}$, associated with effects induced by the heavy KK modes as well as the gauge boson zero mode wavefunction and mass corrections respectively. These functions also depend on the localization of the fermions in the bulk.

In the case of bulk fermions which couple to the Higgs in a relevant way, there may be other important loop corrections to the $T$ parameter. These originate from the breakdown of the custodial symmetry associated with the large top-quark Yukawa coupling and constrain the right-handed top-quark mass parameter to be negative, $c_{t_{R}}<0$, while the value of the left-handed top-quark mass parameter must be smaller than the conformal case value, $c_{Q_{L}^{3}}<$ 0.5. In our work we have chosen values of the fermion mass parameters $c_{f}$ such that the contributions to flavor changing neutral currents and to $R_{b}=\Gamma(Z \rightarrow b \bar{b}) / \Gamma(Z \rightarrow$ hadrons $)$ are suppressed, while the fermion induced contributions to the precision electroweak observables become subdominant.

The result of our analysis shows that, with small but non-vanishing local brane kinetic terms for the gauge fields, one can obtain a model consistent with unfication of couplings and with precision electroweak data with gauge boson KK masses of the order of a few TeV. The bound on the gauge boson KK masses is correlated with the value of the Higgs mass, and a light KK spectrum demands Higgs masses larger than $300 \mathrm{GeV}$. Thus, the RS unified framework provides a scenario which is a novel alternative to 4-dimensional SUSY grand unification, with potentially interesting experimental signatures at the LHC. 


\section{Acknowledgements}

The authors are pleased to acknowledge conversations with K. Agashe and R. Sundrum. Work at ANL is supported in part by the US DOE, Div. of HEP, Contract W-31-109-ENG38. Fermilab is operated by Universities Research Association Inc. under contract no. DEAC02-76CH02000 with the DOE. A. D. is supported by NSF Grants P420D3620414350 and P420D3620434350 and also wants to thank the Theory Division of Fermilab for the kind invitation.

\section{References}

[1] N. Arkani-Hamed, S. Dimopoulos and G. R. Dvali, Phys. Lett. B 429, 263 (1998) arXiv:hep-ph/9803315.

[2] L. Randall and R. Sundrum, Phys. Rev. Lett. 83, 3370 (1999) arXiv:hep-ph/9905221.

[3] A. Pomarol, Phys. Rev. Lett. 85, 4004 (2000) arXiv:hep-ph/0005293. L. Randall and M. D. Schwartz, JHEP 0111, 003 (2001) arXiv:hep-th/0108114; Phys. Rev. Lett. 88, 081801 (2002) arXiv:hep-th/0108115; K. w. Choi, H. D. Kim and Y. W. Kim, JHEP 0211, 033 (2002) arXiv:hep-ph/0202257; arXiv:hep-ph/0207013 K. Agashe, A. Delgado and R. Sundrum, Nucl. Phys. B 643, 172 (2002) arXiv:hep-ph/0206099; R. Contino, P. Creminelli and E. Trincherini, JHEP 0210, 029 (2002) arXiv:hep-th/0208002; A. Falkowski and H. D. Kim, JHEP 0208, 052 (2002) arXiv:hep-ph/0208058; K. w. Choi and I. W. Kim, Phys. Rev. D 67, 045005 (2003) arXiv:hep-th/0208071; L. Randall, Y. Shadmi and N. Weiner, JHEP 0301, 055 (2003) arXiv:hep-th/0208120; A. Lewandowski, M. J. May and R. Sundrum, Phys. Rev. D 67, 024036 (2003) arXiv:hep-th/0209050; K. Agashe and A. Delgado, Phys. Rev. D 67, 046003 (2003) arXiv:hep-th/0209212.

[4] W. D. Goldberger and I. Z. Rothstein, Phys. Rev. Lett. 89, 131601 (2002) arXiv:hep-th/0204160; arXiv:hep-th/0208060; arXiv:hep-ph/0303158

[5] K. Agashe, A. Delgado and R. Sundrum, Annals Phys. 304, 145 (2003) arXiv:hep-ph/0212028. 
[6] W. D. Goldberger, Y. Nomura and D. R. Smith, Phys. Rev. D 67, 075021 (2003) arXiv:hep-ph/0209158.

[7] S. J. Huber, C. A. Lee and Q. Shafi, Phys. Lett. B 531, 112 (2002) arXiv:hep-ph/0111465.

[8] C. Csaki, J. Erlich and J. Terning, Phys. Rev. D 66, 064021 (2002) arXiv:hep-ph/0203034.

[9] H. Davoudiasl, J. L. Hewett and T. G. Rizzo, arXiv:hep-ph/0212279.

[10] M. Carena, E. Pontón, T. M. Tait and C. E. Wagner, arXiv:hep-ph/0212307.

[11] M. Carena, T. M. P. Tait and C. E. M. Wagner, Acta Physica Polonica, B33, 2355 (2002) arXiv:hep-ph/0207056.

[12] A. Manohar and H. Georgi, Nucl. Phys. B 234, 189 (1984); M. A. Luty, Phys. Rev. D 57, 1531 (1998) arXiv:hep-ph/9706235. A. G. Cohen, D. B. Kaplan and A. E. Nelson, Phys. Lett. B 412, 301 (1997) arXiv:hep-ph/9706275; Z. Chacko, M. A. Luty and E. Pontón, JHEP 0007, 036 (2000) arXiv:hep-ph/9909248.

[13] G. Burdman, Phys. Rev. D 66, 076003 (2002) arXiv:hep-ph/0205329.

[14] J. L. Hewett, F. J. Petriello and T. G. Rizzo, JHEP 0209, 030 (2002) arXiv:hep-ph/0203091.

[15] Y. Kawamura, hep-ph/9902423, Prog. Theor. Phys. 103, 613 (2000); hep-ph/0012125, Prog. Theor. Phys. 105, 999 (2001) and hep-ph/0012352, Prog. Theor. Phys. 105, 691 (2001); G Altarelli and F. Feruglio, hep-ph/0102301, Phys. Lett. B 511, 257 (2001); L. Hall and Y. Nomura, hep-ph/0103125, Phys. Rev. D 64, 055003 (2001); A. Hebecker and J. March-Russell, hep-ph/0106166, Nucl. Phys. B 613, 3 (2001); R. Barbieri, L. J. Hall and Y. Nomura, hep-ph/0106190, Phys. Rev. D 66, 045025 (2002).

[16] R. Barbieri, L. J. Hall and Y. Nomura, Phys. Rev. D 63, 105007 (2001) arXiv:hep-ph/0011311.

[17] C. D. Carone, Phys. Rev. D 61, 015008 (2000) arXiv:hep-ph/9907362; A. Delgado, A. Pomarol and M. Quiros, JHEP 0001, 030 (2000) arXiv:hep-ph/9911252; D. E. Kaplan and T. M. Tait, JHEP 0111, 051 (2001) arXiv:hep-ph/0110126; S. J. Huber, arXiv:hep-ph/0303183 
[18] T. Gherghetta and A. Pomarol, Nucl. Phys. B 586, 141 (2000) arXiv:hep-ph/0003129.

[19] M. E. Peskin and T. Takeuchi, Phys. Rev. D 46, 381 (1992).

[20] F. del Aguila, M. Perez-Victoria and J. Santiago, arXiv:hep-ph/0305119.

[21] T. Tait and C. P. Yuan, Phys. Rev. D 63, 014018 (2001) arXiv:hep-ph/0007298.

[22] B. A. Dobrescu and C. T. Hill, Phys. Rev. Lett. 81, 2634 (1998) arXiv:hep-ph/9712319;

R. S. Chivukula and N. Evans, Phys. Lett. B 464, 244 (1999) arXiv:hep-ph/9907414;

M. E. Peskin and J. D. Wells, Phys. Rev. D 64, 093003 (2001) arXiv:hep-ph/0101342;

D. Choudhury, T. M. Tait and C. E. Wagner, Phys. Rev. D 65, 053002 (2002) arXiv:hep-ph/0109097.

[23] http://lephiggs.web.cern.ch/LEPEWWG/. 